\title{
La folie des grandeurs. L'écrivain et la chose littéraire dans les essais de Richard Millet
}

\author{
Martine-Emmanuelle Lapointe \\ Université de Montréal
}

Comme en témoigne un récent dossier de la revue Acta Fabula consacré aux "Tombeaux pour la littérature», les discours critiques sur la mort de la culture littéraire se multiplient à l'époque contemporaine. Ces derniers constitueraient même, selon Alexandre Gefen, "un objet polémique communément partageable» (2009). Dominique Maingueneau (2006), William Marx (2005) et Tzvetan Todorov (2006), notamment, ont consacré leurs récents ouvrages à la déconsidération dont la littérature serait aujourd'hui la victime. Leurs études tendent à historiciser les discours sur la fin de la littérature et, dans une certaine mesure, à alerter les lecteurs, les professeurs et les 
critiques sur les dangers que comporte la fragilisation de la culture littéraire. Il s'agit surtout, chez les trois auteurs, de dépasser les bilans catastrophistes et mortifères pour mieux cerner les causes de la dévalorisation de la littérature. Il en va tout autrement dans les derniers essais de Richard Millet qui font eux aussi état de la disparition de la littérature à l'époque contemporaine, mais sur le mode de la complainte et de la déploration. Pour Millet, âpre contempteur de la culture actuelle, le contemporain évoluerait dans un monde désenchanté, peuplé des spectres d'un passé glorieux. Il errerait, ne vivrait qu'avec son temps, amnésique et aveugle, dépourvu d'idéal et de grandeur. Bien triste sire, le contemporain, en somme, ne serait que le contemporain de luimême.

Ces constats catastrophistes se trouvent plus précisément dans Harcèlement littéraire, livre d'entretiens accordés par l'auteur à Delphine Descaves et Thierry Cecille, et dans le court essai Le Dernier écrivain, parus tous deux en 2005, ainsi que dans les très polémiques Désenchantement de la littérature et L'Opprobre, publiés respectivement en 2007 et en 2008. Ces ouvrages constituent un ensemble cohérent dans lequel l'auteur dénonce la vacuité de la littérature contemporaine en se revendiquant des traditions humaniste et chrétienne de l'Occident. Rompant avec le ton personnel et inquiet des textes rassemblés dans les trois tomes du Sentiment de la langue, Millet n'hésite pas à y entrelacer lectures élogieuses d'œuvres du passé, critiques acerbes de la littérature contemporaine et commentaires sociopolitiques radicaux.

Il va sans dire que certains de ces textes, Désenchantement de la littérature plus que les autres, ont été 
très mal reçus par la critique française, qui a accusé leur auteur de xénophobie. Pour mieux dénoncer la rectitude politique et le "totalitarisme mou », l'auteur s'en est pris avec virulence aux théories du métissage, du multiculturalisme et de l'hybridation en leur opposant les concepts problématiques de la vérité et de la pureté. Dans un débat qui réunissait Millet et Sollers, paru en 2007 dans Le Magazine littéraire, Sollers a d'ailleurs affirmé :

Je crois que Richard Millet a eu un tort, celui de mêler à ses considérations sur la littérature des idées politiques, et des idées politiquement incorrectes. Elles ont permis à l'opinion, surtout l'opinion militante, se voulant extrêmement engagée, de l'accuser, avec des mots injurieux, d'être révisionniste et d'avoir écrit une immondice. (2007, p. 92-93)

Comme le note Sollers, qui n'hésite pas à qualifier la réception de Désenchantement de la littérature d'«inquisitoriale» et de « stalinienne» (p. 93), les constats catastrophistes de Millet ont été sévèrement critiqués, car ils débordaient le contexte littéraire et empiétaient sur le territoire de la politique contemporaine. En s'en prenant aux « diktats du politiquement correct» (p. 93) et en défendant la préservation de la langue et des traditions françaises, Millet a endossé — peut-être malgré lui ${ }^{1}$ - le rôle du redresseur de torts et s'est ainsi vu isolé du milieu littéraire parisien ${ }^{2}$. Au fil des textes parus en marge de

\footnotetext{
1 Dans l'entrevue accordée au Magazine littéraire, Sollers reproche à Millet de lier trop étroitement la littérature et la société. Or Millet prétend faire cavalier seul, corroborant même les propos suivants de Sollers : la littérature est une « aventure parfaitement individuelle, réfractaire, singulière et qui résiste à tout » (p. 93).

2 Dans «Droit de réponse de la gestapo à Richard Millet», publié sur son blogue, Pierre Assouline (2007) a bien fait ressortir les contradictions de Désenchantement de la littérature : «Le malentendu est tragique : il croit qu'il est dans la vérité à mesure que le nombre de ses ennemis augmente. Encore faut-il préciser que les ennemis en question ne sont que des critiques que sa
} 
l'œuvre romanesque, l'auteur se présente en effet comme le dernier d'une grande lignée, héritier d'une tradition moribonde à laquelle nul ne semble plus vouloir s'attacher. Une telle posture s'avère quasi intenable, car elle repose sur une argumentation manichéenne multipliant les oppositions binaires, les constats fatalistes et les dénégations.

Afin de mieux réfléchir à la construction de cette figure de l'écrivain, j'analyserai ici l'un des paradoxes fondateurs de l'argumentation de Richard Millet. Se retirant dans une sorte de retraite splendide, qui lui conférerait le rôle de l'émissaire éclairé, Millet se dit « déprogrammé », indifférent aux diktats et aux langages qui dominent les discours sociaux et culturels de son époque. Mais cette déprogrammation présumée ne peut tenir puisque l'auteur programme à même son argumentation les critiques de ses détracteurs, use d'une rhétorique qui se forme littéralement à partir de l'ethos contemporain. Même s'il refuse haut et fort de jouer le jeu de la politique, Millet se retrouve dans l'arène, écartelé entre l'idéalisme du «dernier écrivain » et l'hyperréalisme du pamphlétaire bien au fait des problèmes de son époque.

\section{De la déprogrammation}

C'est dans Désenchantement de la littérature que Millet définit la notion de déprogrammation :

Déprogrammation, plutôt que censure ou interdiction : telle pourrait être une autre définition de la littérature, négative celle-là, son ordalie ténébreuse, et indissociable du sentiment d'encombrement de soi que peut donner à l'écrivain sa

conception légèrement paranoïaque de la vie vécue comme une guerre civile permanente transforme en ennemis. » 
notoriété. Mieux que le silence et le retrait : à mille lieues de la multitude piaillante des auteurs, l'écrivain serait celui qui, devançant inlassablement les condamnations émises par le nouvel ordre moral, se déprogramme lui-même, se voue à l'échec comme à une forme de salut. (2007, p. 21)

La déprogrammation se substituerait au nouvel ordre moral auquel Millet associe l'idée d'une censure idéologique, thème également présent dans Harcèlement littéraire. Les termes « ordalie» et «salut», issus du lexique chrétien, renforcent l'effet de la déprogrammation qui, plus qu'une retraite silencieuse, constitue à la fois un sacrifice, une épreuve et une forme d'élection. Plus loin, s'inspirant d'une citation de Peter Handke, Millet ajoute qu'il est le troisième homme, ni coupable ni héros, "l'improgrammable, celui qui s'invente dans le paradoxe de son propre retrait, eût-il le bruit du monde pour destin de son langage » (p. 22). Bien qu'il reconnaisse la nature paradoxale d'une telle situation - définie par la négative et dégagée en apparence de ses ancrages sociaux et politiques l'auteur confère à la déprogrammation la valeur d'un sacre. Millet souhaite s'éloigner de «la multitude piaillante des auteurs ». L'emploi du mot « auteur » n'est pas fortuit; l'écrivain se distingue de l'auteur, car il accueille l'échec. Plus encore, il se refuse aux usages mondains, aux langages communs et aux credo du «nouvel ordre moral » Dès l'incipit du Dernier écrivain, Millet affirme ne pas être de son temps, se dit « apatride », prétend trouver son lieu dans l'écart, le décalage, le refus (2004, p. 11).

La langue et le style constituent des critères d'élection ou de discrimination permettant de tracer une frontière nette entre les figures de l'auteur et de l'écrivain. Millet s'en explique d'ailleurs assez longuement dans le recueil d'entretiens 
Harcèlement littéraire. Son isolement ne serait pas « une forme d'asocialité heureuse » (2005, p. 32), propre à l'artiste maudit, mais reposerait sur son mépris confirmé des œuvres de ses contemporains. Ceux-ci ne sauraient plus écrire, trufferaient leurs textes d'erreurs, de solécismes et de barbarismes : "Ce qui gêne, affirme-t-il, c'est l'incertitude syntaxique, l'inharmonie, la condition postmoderne de la langue » (p. 35); «cette approximation, cette mollesse [...]. Elles reflètent bien notre époque, molle, veule, obsédée par le ludique, le "sympa", l'édulcoré, l'éphémère, l'insignifiant» (p. 36). Cette dernière affirmation, qui transforme la langue de l'écrivain en reflet d'une condition sociologique anémique et défaillante, traverse presque tous les écrits essayistiques et pamphlétaires de l'auteur. La maîtrise de la langue devient en quelque sorte le symbole de l'appartenance à une forme d'aristocratie littéraire et culturelle, laquelle s'opposerait en tous points à la démocratie états-unienne et à son culte des droits de l'homme.

\section{L'idiot et le martyr}

La fratrie à laquelle Millet dit appartenir est une communauté d'exception qui rassemble ceux qui échappent aux normes, qu'ils soient des êtres rares, des génies méconnus, des « renards solitaires » (2007, p. 12), pour reprendre l'expression de l'auteur, ou des exclus dépossédés de leurs lieux propres : « Je suis donc le frère impossible de tout juif tchèque, le gant retourné, le provincial sans terre qui prétend à l'universel, refuse de confondre minoritaire et infantilisme, et répugne à incanter les mots d'ordre du Bien» (2004, p. 20). Parmi les figures invoquées par l'auteur, ce sont celles de l'idiot et du martyr qui incarnent de la manière la plus éloquente la double 
nature de cette communauté improbable. Elles deviennent même, sous la plume de Millet, les représentantes d'un ostracisme social injustifié.

$\mathrm{Si}$ elles traversent les essais et les pamphlets, les représentations les plus évocatrices de la figure de l'idiot se trouvent dans les fictions de l'auteur. Inspirées des personnages de Faulkner et de Dostoïevski, elles y empruntent les traits d'André Pythre, le dernier fils d'une lignée maudite, de Pierre-Marie Lavolps, du Renard dans le nom, ou encore de Lucie Piale, l'innocente à l'éternel sourire de L'Amour des trois sœurs Piale. Les trois personnages, mais il en est bien d'autres, sont dotés d'une beauté qui les marginalise et les sauve à la fois, et appartiennent par là même à un monde autre. Souvent retranchés dans leur silence, qui peut passer pour une forme de béatitude, ils inquiètent les habitants de Siom, qui les confondent tantôt avec les anges, tantôt avec les démons. Dans ses essais, Millet établit une analogie entre ces figures d'humbles et celle de l'écrivain véritable - l'adjectif "véritable» est de l'auteur - qu'il compare même aux «chrétiens des catacombes» (2005, p. 63). Comme les innocents, dont la parole est souvent mésestimée, l'écrivain " ne s'en remet plus à l'ordre du monde », il se livre plutôt à « un non-savoir qui est la condition d'une connaissance supérieure » (2007, p. 28). L'exemple le plus révélateur de cette relecture chrétienne du rôle de l'écrivain se trouve au tout début de L'Opprobre, au sous-titre évocateur d'Essai de démonologie. Dans ce recueil de fragments rappelant le propos du Dernier écrivain et de Désenchantement de la littérature, Millet se présente littéralement comme un revenant :

C'est donc un mort qui parle, un suicidé du milieu littéraire. Je ne suis pas le premier; je ne serai pas le dernier. Et je reviens 
vous hanter, belles âmes qui pensez m'avoir tué. L'outre-tombe d'où je parle est en réalité le lieu le plus vivant, au sein de l'inversion actuelle de toutes les valeurs : le vivant contre le vrai devenu non seulement un moment du faux mais le faux luimême. Ma voix est donc celle de la vérité. Je n'écrirais pas si je ne me maintenais pas à cette hauteur, avec tout à la fois l'aplomb des humbles, l'intransigeance des orgueilleux, l'opiniâtreté de ceux qui sont toujours en chemin. (2008, p. 12)

Depuis la publication de Désenchantement de la littérature, qualifiée de geste suicidaire par certains critiques ${ }^{3}$, Millet se dit mort à l'existence littéraire. Mais cette mort, en témoigne la citation, est pour lui le lieu du vrai, voire le seul lieu possible dans le contexte contemporain, la situation la plus anachronique qui soit. La scène qui ouvre L'Opprobre, si on peut la qualifier ainsi, en est révélatrice à plusieurs égards. Millet, d'une part, s'y fait le représentant d'une lignée de suicidés littéraires - au sens figuré - , dont il livrera les noms dans un fragment ultérieur. Cette liste donne corps à la communauté improbable des écrivains chers à Millet :

Proust, Kafka, Musil, Joyce, morts dans l'inachevé ou l'inachevable; Bernanos, Sartre, Malraux, Blanchot, abandonnant le roman après la Seconde Guerre mondiale; Woolf, Drieu la Rochelle, Hemingway, Kawabata, Mishima, Márai se suicidant; Faulkner, Giono, Nabokov, Beckett, Duras, Simon, quelques autres encore faisant survivre le corps romanesque jusqu'à l'extrême du possible. (2008, p. 33)

La communauté imaginaire ici recréée rassemble des prosateurs du $\mathrm{XX}^{\mathrm{e}}$ siècle, dont les styles, les appartenances culturelles et les orientations politiques diffèrent. L'idée

\footnotetext{
${ }^{3} \mathrm{Au}$ sujet de la polémique déclenchée par la publication de Désenchantement de la littérature et prolongée par celle de L'Opprobre, voir notamment les articles de Kéchichian (2007), Launet (2008), Sebag (2008), Macé-Scaron (2008), Liger (2008) et Rüf (2009).
} 
directrice demeure celle d'une certaine inadéquation à la vie sociale, voire celle d'un sentiment d'insatisfaction générale qui conduirait au refus des codes dominants et, dans les cas les plus radicaux, à la mort volontaire. Il va sans dire qu'un tel rassemblement repose sur des effets de lecture qu'une analyse plus approfondie contredirait. Que penser, par exemple, de la présence de Jean-Paul Sartre, qui n'a jamais nié l'existence de relations objectives entre la littérature et la société? Qu'en est-il du rapprochement d'écrivains aux idéologies et aux esthétiques radicalement opposées? Les résistants côtoient les collaborateurs; les provincialistes sont confondus avec les modernistes et les Nouveaux Romanciers; les frontières entre les corpus nationaux sont abolies. Tout se passe comme si Millet souhaitait se débarrasser des ancrages et des appartenances pour mieux défendre une conception parfaitement anachronique de la littérature, ce qui n'est pas sans faire écho à la posture radicale qu'il endosse. Au sein de sa bibliothèque singulière néanmoins, quelques idées directrices demeurent. L'inachèvement, le suicide et la survivance renvoient aux tensions entre la vie et la mort, entre le commencement et la fin, l'auteur n'hésitant guère à recourir à de grandes antinomies symboliques pour mieux soutenir son propos.

L'écrivain véritable, si l'on se fie au portrait qu'en offre l'auteur, est donc un martyr, un "suicidé de la société » pour citer le fameux texte qu'Artaud consacra à Van Gogh en 1947 et qui, à l'instar des interprétations de Millet, procède d'une inversion radicale des valeurs communément admises. Pour Artaud, rappelons-le, "l'aliéné est aussi un homme que la société n'a pas voulu entendre et qu'elle a voulu empêcher d'émettre d'insupportables vérités » (2001, p.17). Rien 
d'étonnant à ce que Millet s'identifie à la figure d'Artaud, en qui il voit « une forme de sainteté, sans l'appareil religieux » et une manière de vivre sa foi «dans son corps jusqu'à l'extrême du possible» (2005, p. 62). En plus de la mort à l'existence littéraire, fondement même d'une communauté de la marge et de la vérité, plusieurs passages de L'Opprobre, dont la scène d'ouverture, mettent clairement en œuvre une rhétorique inspirée de la tradition chrétienne. L'écrivain est porteur d'une parole vraie, venue d'outre-tombe, ce qui rappelle à la fois les figures du Christ et de Lazare ; il se maintient à cette hauteur, précise-t-il, et se soucie peu de l'opprobre dont il sera encore une fois la victime volontaire. Tel le martyr donc, Millet se dit prêt à aller jusqu'au sacrifice de sa vie pour défendre ses valeurs et ses convictions.

Entre les figures de l'idiot et du martyr s'esquisse ainsi un réseau de correspondances reposant sur des motifs récurrents, qu'il s'agisse de l'ostracisme social, du mystère d'une vérité inaccessible à la majorité, mais surtout de l'incorporation, sous des formes parfois extrêmes, de la parole. Dans un article consacré à la rhétorique de l'idiot chez Faulkner, Hébert et Jacob, Julie Ouellet montre bien comment la figure de l'idiot s'accompagne souvent d'un certain messianisme :

Le langage de Jésus semble si simple : tout comme nos idiots, il prononce sa parole en citant les choses terrestres à titre d'explication. Cependant, l'objet de son discours (la foi pour Jésus, les passions humaines dans le cas de l'idiot) se situe à l'extérieur du monde concret. Il s'agit de l'intouchable. Encore pis, de l'insoutenable. Toutefois, Jésus parvient à soutenir l'insoutenable grâce à son argumentation qui emprunte la voie herméneutique et, comme le poète et l'idiot qui n'affirment rien, il ne peut donc mentir. (2001, p. 183) 
En prétendant défendre la vérité, en déclarant ses propos insoutenables, Millet se réfugie lui aussi dans le domaine de l'intouchable. L'Opprobre est sans doute le texte qui radicalise le plus clairement cette posture, car l'auteur y compile des fragments qui empruntent parfois les contours de l'aphorisme, de la citation biblique, de la sentence. L'absence de ligne directrice, de syntaxe si l'on peut dire, leur attribue la valeur d'une réflexion apparemment libre et spontanée, en dehors des règles figées de la rhétorique ou de l'argumentation classique. Ce parti pris formel confère également une assise matérielle à une pensée qui se revendique à la fois de la subjectivité la plus radicale - Millet avoue préférer la narration à la première personne - et de l'inachèvement. L'auteur s'enfermerait ainsi dans une réflexivité absolue qui conduirait au déploiement d'une pensée ouvertement contradictoire et, fort paradoxalement, à la dissolution de son ego, son «je » anachronique acquérant dès lors une valeur impersonnelle.

\section{Impossible décontextualisation}

Suivant cette logique, la déprogrammation de l'écrivain serait une manière de se détacher du monde tout en $\mathrm{y}$ demeurant attaché, de se tenir entre la vie et la mort, dans une sorte de tiers-lieu. En somme, écrire consisterait à « en appeler à "l'êtreen-commun" par la tension paradoxale et extrême d'une désolidarisation » (2008, p. 71). Cette désolidarisation tendrait même vers une forme d'impersonnalité. Millet serait l'« écrivain sans figure, ne ressortissant à nul des particularismes raciaux, ethniques, religieux, sexuels, linguistiques ou physiques qui régissent la spécialisation scripturaire» (2004, p. 27), il ne serait «que question, béance d'un questionnement sans fin » 
(2008, p. 18); mieux, il ne devrait jamais «être pris au pied de la lettre journalistique ou politique» (p.17). Mais ces précautions rhétoriques ne convainquent guère. Le discours de Millet, même inspiré par les écrits de Blanchot ${ }^{4}$, ne parvient jamais réellement à se dépouiller de l'expressivité individuelle, des attributs d'une personnalité bien identifiable. Nous sommes loin de l'anonymat ou de la disparition élocutoire du poète. L'auteur du Désenchantement de la littérature est toujours en situation, bien ancré dans son époque. Peut-être est-ce un truisme, mais penser contre, c'est aussi penser avec, comme le suppose implicitement le sens premier du mot «contemporain». Or les lieux contre lesquels et à partir desquels Millet pense sont le milieu littéraire parisien, la littérature de l'extrême-contemporain, la situation de l'enseignement secondaire en France $^{5}$ et, bien sûr, le «totalitarisme mou» qui va de pair avec le mouvement de déchristianisation du monde occidental. Il ne suffit pas d'affirmer que «le langage de la littérature n'est pas sociologique : il est l'écart absolu » (2005, p. 39) pour échapper à l'emprise du sociologique. Une telle posture s'avère intenable - tout comme son envers d'ailleurs -, car elle omet de considérer les incontournables médiations entre l'œuvre et son milieu, aussi discrètes soient-elles. Les essais de Millet, comme certains de ses romans, ne font absolument pas les morts sur le plan politique, ils sont au contraire nourris, pétris, modelés par

\footnotetext{
4 Dans Le Dernier écrivain, Millet évoque notamment la fatigue, le désespoir ainsi que «la fraîcheur souveraine de l'écart» $(2004$, p. 32) que lui aurait enseignés Blanchot.

${ }^{5}$ Les critiques les plus explicites à l'égard du système d'éducation français se trouvent dans Harcèlement littéraire. Expliquant pourquoi il a quitté l'enseignement, Millet déplore, entre autres, la disparition de la conscience linguistique et de la culture générale chez les élèves (2005, p. 173-174).
} 
les discours socioculturels contemporains au point de ne pouvoir être réellement compris par un lectorat qui n'entendrait rien aux discours de la Cité (ou de la métropole) 6 . L'Opprobre s'avère particulièrement éclairant à cet égard. Millet s'en prend au Démon, figure par excellence de l'ennemi condamné à « [l']ignorance volontaire, [la] lâcheté, [la] servilité, [la] grégarité, [le] moralisme: tous les attributs du multiculturalisme » (p. 22).

La preuve de cette impossible sortie du sociologique se trouve notamment dans les essais mêmes de l'auteur, qui programment les critiques de leurs potentiels détracteurs. Une part importante des textes est ainsi réservée à la réfutation des arguments passés ou à venir des «ennemis » de Millet. Mieux, en contrepoint de la figure de l'écrivain véritable, s'impose celle de l'auteur à succès, qui écrit comme il parle et qui pense comme la majorité, c'est-à-dire mal. Dans Harcèlement littéraire, les cibles sont clairement identifiées : Libération et Télérama sont qualifiés de "prescripteurs », de "générateurs de doxa », de «laboratoires du politiquement correct» (p. 50); les écrivains de Minuit produisent «des entrechats sur les genres dits mineurs » et ceux de Verdier sont, même s'ils «jouent sur l'image d'une littérature exigeante, difficile, marginale », soumis aux contraintes du système et tout aussi consensuels que les auteurs publiés par les grandes maisons d'édition (p.67). Seuls quelques contemporains échappent au

\footnotetext{
6 À titre d'exemple, l'une des pages de L'Opprobre ne pourrait être déchiffrée par un lecteur peu au fait de la réception défavorable de Désenchantement de la littérature. Constituée de citations non référencées, elle se présente littéralement sous la forme d'un collage : «je ferais de la politique, je serais un "pseudoprophète égaré dans ses vaticinations idéologiques", une "langue de pute", un "écrivain en perte d'altitude" » (2008, p. 13), pour ne citer que les premiers mots de la longue énumération de Millet.
} 
courroux de Millet: Quignard, Bergounioux, Michon, sans oublier feu Blanchot. À la littérature pamphlétaire, les derniers textes de Millet empruntent donc la volonté "d'abattre un adversaire [et de] défendre une cause » (Croquette), adoptant une rhétorique du combat et de la riposte.

En témoigne l'importance du mot "opprobre » qui, en plus de donner son titre au dernier essai de l'auteur, traverse également les pages du Dernier écrivain et de Désenchantement de la littérature. À la deuxième page du Dernier écrivain, Millet déclare: "Non que je veuille maudire plus que de raison une époque qui est, elle, toujours sur le point de me réprouver : ayant connu certaines formes d'opprobre, je lui suis même reconnaissant de me montrer le visage d'un futur où je ne serai pas » $(2004$, p. 12). Les premières pages du Désenchantement de la littérature résonnent de manière similaire. Pour décrire l'opprobre qui le menace, l'auteur se projette dans le sort injuste d'un autre écrivain :

Peter Handke, si exemplairement écrivain, car peu présent physiquement, sauf là où il ne devrait pas se trouver, diront les chiens de garde, et où il est vu (c'est-à-dire vilipendé au lieu d'être lu), aux funérailles de Slobodan Milosevic, en Serbie, pays qui n'en finit plus d'expier, et de subir, après des semaines de bombardements américano-européens, la guerre langagière des démocraties occidentales, une sorte d'opprobre. Un écrivain ne témoigne que de lui-même et ce témoignage doit être écouté plutôt que jugé d'avance. (p. 18)

Celui qui prétendait être indifférent à son époque prouve, par ces affirmations, qu'il demeure sur le qui-vive, prêt à recevoir répliques violentes et injustes. En s'identifiant à la figure de Handke, écrivain respecté et reconnu qui aurait commis l'erreur de prendre le mauvais parti politique, Millet se pare d'une sorte de bouclier symbolique lui permettant de contrer les ripostes 
éventuelles. Rappelons que les critiques les plus virulentes des essais de Millet paraissent à la suite de Désenchantement de la littérature et de L'Opprobre. C'est dire, encore une fois, que Millet était conscient de la portée de ses critiques apocalyptiques et de ses commentaires désobligeants sur la culture contemporaine. Programmé, il était solidaire malgré lui de son époque.

La notion de "totalitarisme mou», tout autant que la médiocrité générale de la langue écrite et parlée, constitue l'un des sujets de prédilection de Millet. C'est, semble-t-il, lorsqu'il traite de cet aspect de l'ethos contemporain que l'auteur donne le plus radicalement dans la rhétorique pamphlétaire. Il en arrive par là même à saboter son entreprise de déprogrammation. Évoqué dans les quatre textes étudiés, ce fameux «totalitarisme mou» est clairement défini dans Harcèlement littéraire :

Comment un totalitarisme s'établit-il? Sur une forme de consensus né d'un processus de simplification extrême. Tous les totalitarismes sont nés d'idées extrêmement simples, voire simplistes, et se sont imposés, autant que par les armes, par un usage populiste de la langue et de mythes nationaux détournés. Les deux vecteurs de ce totalitarisme mou sont la télévision, la presse, et un système scolaire qui ne transmet plus que les mots d'ordre de l'Empire du Bien. (p. 171)

Jusqu'ici rien d'alarmant. La définition repose sur une analyse plutôt descriptive, à des lieues de la hargne et de l'anathème. Mais quel est cet Empire du Bien ? À quelle réalité s'attache-til ? Quels sont ses fondements idéologiques ? Comme l'explique Millet dans Désenchantement de la littérature, le Bien serait désormais aux antipodes de l'ancienne verticalité européenne 
- blanche, hétérosexuelle et chrétienne, précise-t-il - et serait le produit d'une décadence :

L'effondrement du vertical au profit de l'horizontal n'est pas seulement emblématique de la fin du christianisme: il est l'actualisation d'une dévalorisation générale. De sorte que dans un monde qui aurait perdu le sens même du sens, un monde où tout se vaudrait (le minuscule le grand, le bas le haut, le déviant la norme, le mélange la pureté, l'exception la loi, selon la jurisprudence perpétuelle de l'individuel). (p. 25)

Ces deux définitions annoncent les dérives - sur l'Islam, le métissage racial et la culture états-unienne - auxquelles se livre ultérieurement l'auteur dans les pages de Désenchantement de la littérature et de L'Opprobre. Ces pages ne relèvent plus de l'analyse ou du débat intellectuel, mais sont le fruit du ressentiment. Et c'est sans doute parce qu'elles sont si étroitement rattachées à l'expression revancharde d'une subjectivité que les affirmations de Millet passent si mal. Mépris, dégoût, humeurs transparaissent dans la prose, certes élégante et maîtrisée, de l'auteur. Les vagues échos à la Société du Spectacle ${ }^{7}$ de Guy Debord, ouvrage dénonçant un certain totalitarisme médiatique, y sont dépouillés de leur caractère d'abstraction. À des concepts et à des constats généraux Millet associe, souvent acrimonieusement, des noms propres, des lieux géographiques, des religions qu'il diabolise. Il se retrouve bien loin, en somme, de la déprogrammation, du silence et de la retraite que semblait promettre la littérature.

7 Dans Désenchantement de la littérature, Millet évoque le «Spectacle démocratique » (2007, p. 19) auquel est lié le totalitarisme mou. Plus loin, il prise la grande langue de Debord, note qu'elle «a quelque chose de superbement militaire» (p. 58). C'est cependant dans Harcèlement littéraire que les références à La Société du Spectacle sont les plus explicites, l'auteur comparant la société contemporaine à un système "où, comme dit Debord, le vrai n'est qu'un moment du faux » $(2005$, p. 51). 
Nullement déprogrammé, Richard Millet est incapable de cette désolidarisation dont il se prétend le porte-parole. Au contraire, il se retrouve dans l'arène, il s'y jette presque candidement en espérant trouver un lecteur qui lui ressemble, un frère, un semblable. Et peut-être est-ce là que réside réellement le problème de ses derniers essais. Alors qu'ils devaient donner lieu à de véritables débats sur la place publique, ils sont demeurés lettres mortes, faute d'interlocuteurs prêts à recevoir les propositions catastrophistes de leur auteur. Ils n'étaient tout compte fait que soliloques rédigés par un écrivain en mal de lectorat - peut-être le dernier d'une lignée nihiliste et suicidaire. Il se trouvera bien un lecteur ou deux, auteurs de lettres ouvertes admirablement rédigées, pour le défendre. Ceux-ci n'hésiteront guère à déplorer le piètre état du système d'éducation français, la médiocrité du journalisme et l'absolue vacuité du roman contemporain. Mais ils le feront en empruntant les arguments et la posture de Millet, eux aussi pris dans les rouages, non pas d'un totalitarisme mou, mais d'un système de pensée relativement prévisible.

Comment en effet engager le débat lorsque l'on emprunte une rhétorique volontairement manichéenne, démonologique, hostile à la nuance et au compromis? L'idiot et le martyr ne sont-ils pas des figures qui ne tolèrent nullement les demi-mesures? L'un s'enferme dans son langage, l'autre dans sa foi, aucun ne pouvant ou ne souhaitant en déroger. Tous deux se tiennent, en somme, dans l'écart absolu, en marge des codes et des appartenances d'autrui. En revanche, le dernier écrivain ne saurait être tout à fait sourd aux rumeurs médiatiques qui, même décriées, constituent l'arrière-monde de sa réflexion. Il devra affronter, sans doute malgré lui, la « démonialité de [ses] ennemis » (2008, p. 22) et les errements de sa Cité. 


\section{Bibliographie}

Assouline, Pierre (2007). " Droit de réponse de la gestapo à Richard Millet», La République des livres, $<$ http://passouline.blog.lemonde.fr/2007/09/07/droit-dereponse-de-la-gestapo-a-richard-millet/>

ARTAUD, Antonin (2001 [1947]). Van Gogh, le suicidé de la société, Paris, Gallimard, coll. «L'imaginaire».

Croquette, Bernard. «Pamphlet», dans Encyclopaedia Universalis, $<$ www.universalisedu.com/article2.php?napp=\&nref=T301828>

GEFEN, Alexandre (2009). « Ma fin est mon commencement : les discours critiques sur la fin de la littérature », $L H T, \mathrm{n} \times 6$, 10 juin, <http://www.fabula.org/lht/6/dossier/118-gefen>

KÉCHICHIAN, Patrick (2007). «Millet prêche dans le désert», Le Monde des livres, 19 octobre, p. LIV7.

LAUNET, Édouard (2008). « Haut-le-cœur », Libération, nº 8316, 31 janvier, p. 6.

LIGER, Baptiste (2008). «Le masque du démon », Lire, no 365, mai, p. 50-51.

MACÉ-SCARON, Joseph (2008). Marianne, no 571, 5 avril, p. 80.

Maingueneau, Dominique (2006). Contre Saint Proust ou la fin de la littérature, Paris, Belin.

MARX, William (2005). L'Adieu à la littérature, Paris, Minuit. 
MILLET, Richard (2003 [I : 1986 ; II : 1990 ; III : 2003]). Le Sentiment de la langue I-II-III, Paris, La Table Ronde.

- (2004). Le Dernier écrivain, Fontfroide-le-haut, Fata Morgana.

- (2005). Harcèlement littéraire, entretiens avec Delphine Descaves et Thierry Cecille, Paris, Gallimard.

- (2007). Désenchantement de la littérature, Paris, Gallimard.

- (2008). L'Opprobre, Paris, Gallimard.

OuELLET, Julie (2001). "La rhétorique de l'idiot», Études littéraires, vol. 33, no 2, p. 169-185.

RÜF, Isabelle (2009). "Colères d'écrivains », Le Temps, 9 mai, p. 1.

SEBAG, Albert (2008). «Qui a peur de Richard Millet? », Le Point, no 1855, 3 avril, p. 161.

SOLLERS, Philippe (2007). "Quel avenir pour la littérature », Le Magazine littéraire, no 470, décembre, p. 92-93.

Todorov, Tzvetan (2006). La Littérature en péril, Paris, Flammarion.

\section{Résumé}

Dans la série d'essais et d'entretiens qu'il publie entre 2005 et 2008, Richard Millet fait état de l'appauvrissement de la littérature contemporaine sur le mode de la complainte et de la déploration. Au fil des textes parus en marge de son œuvre romanesque, l'auteur se présente comme le dernier d'une 
grande lignée, héritier d'une tradition moribonde à laquelle nul ne semble plus vouloir s'attacher. L'analyse de la figure de l'écrivain "déprogrammé », indifférent aux diktats et aux discours sociaux de son époque, permet de mettre au jour les paradoxes de l'argumentation de l'auteur. Refusant haut et fort de jouer le jeu de la politique, Millet se retrouve néanmoins dans l'arène, écartelé entre l'idéalisme du « dernier écrivain » et l'hyperréalisme du pamphlétaire.

\begin{abstract}
In his essays and interviews published between 2005 and 2008, Richard Millet puts forward the impoverishment of contemporary literature by lamenting and deploring. On the margins of his novels, the author uses his writings to introduce himself as the last one of a great line, heir of a dying tradition no one really wants to belong to any more. The analysis of the « deprogrammed » writer's figure, indifferent to the diktats and social discourses of his time, will bring to light the paradoxes of the author's argumentation. By refusing categorically to play the political game, Millet nonetheless finds himself inside the arena, torn between the idealism of «the last writer » and the hyper-realism of the lampoonist.
\end{abstract}

